\title{
Proposta de requisitos essenciais baseada em rankings internacionais de ensino superior e empreendedorismo
}

DOI: 10.37702/2175-957X.COBENGE.2021.3543

Carolina Maia dos Santos - cmaias@ymail.com

Centro Federal de Educação Tecnológica Celso Suckow da Fonseca

Avenida Oliveira Belo 01

21221-300 - Rio de Janeiro - RJ

Diego Rizzotto Rossetto - diegorossetto@utfpr.edu.br

Universidade Tecnológica Federal do Paraná

Rua Altevir Turra 301

85505-434 - Pato Branco - PR

sonia marise salles carvalho - smarises1960@gmail.com

Universidade de Brasília

condominio RK antares quadra a casa 1

73252-200 - Brasilia - DF

Elzo Alves Aranha - eaaranha@unifei.edu.br

Universidade Federal de Itajubá

Av. BPS 1303

37500-903 - ITAJUBA - MG

Resumo: As exigências profissionais impostas aos engenheiros estão sofrendo mudanças. Por isso, as Diretrizes Curriculares Nacionais de 2019 estabelecem uma formação mais alinhada com as demandas atuais e concedem mais relevância à educação empreendedora nos cursos brasileiros. Este cenário, também oriundo do movimento de globalização, fez surgir alguns modelos de classificação conhecidos como rankings, inclusive, para a educação e o empreendedorismo. Porém, há uma lacuna na literatura brasileira de educação em engenharia sobre este assunto que faz emergir diversas questões. Nesta direção, a presente pesquisa é norteada por: Quais são os requisitos essenciais que estão relacionados com os principais rankings internacionais e são capazes de explicitar a educação em engenharia nas universidades? O objetivo da pesquisa é duplo: a) identificar e explorar os requisitos que estão presentes nos principais rankings internacionais selecionados nesta pesquisa; b) analisar as interfaces entre os requisitos explorados e, com 
base nessas relações, propor um conjunto de requisitos essenciais. Para isso, foi realizada uma pesquisa exploratória, descritiva e explicativa considerando quatro rankings internacionais. Com base na investigação destes modelos, foi possível chegar a uma proposta de requisitos essenciais. Dessa forma, este estudo inova ao apresentar um modelo que fornece uma maior dimensão de análise e ,por outro lado, aponta não ter sido possível identificar a educação empreendedora como requisito essencial nos rankings analisados. Os resultados encontrados evidenciam que, apesar da existência de diferentes rankings com variados objetivos e distintos objetos a serem avaliados, parece haver uma base comum de requisitos entre eles.

Palavras-chave: Educação em Engenharia; Rankings; Educação Empreendedora; Educação Superior; Avaliação 


\section{PROPOSTA DE REQUISITOS ESSENCIAIS BASEADA EM RANKINGS INTERNACIONAIS DE ENSINO SUPERIOR E EMPREENDEDORISMO}

\section{INTRODUÇÃO}

Um longo processo de evolução se desenvolveu desde o início do ensino de engenharia no Brasil em 1792 (OLIVEIRA et al., 2013). Atualmente, as exigências impostas ao engenheiro do século XXI estão sofrendo mudanças em decorrência das profundas modificações do mercado de trabalho e da sociedade, com a qualificação do engenheiro tendo que acompanhar as tendências tecnológicas, econômicas e sociais (SILVA; SANTOS; SOUZA, 2019).

Nos últimos anos, o Brasil não avançou em relação aos países mais competitivos, de acordo com o levantamento do Fórum Econômico Mundial (DIÁLOGOS SETORIAIS UNIÃO EUROPEIA - BRASIL, 2018). O índice analisa o conjunto de políticas e práticas que permitem um país desenvolver, atrair e capacitar o capital humano que contribui para a produtividade e prosperidade. Em 2020, o Brasil aparece na $80^{\circ}$ posição do ranking geral entre 132 nações. Já em relação ao grupo da América Latina e do Caribe, o Brasil encontrase na 11ª posição, tendo o Chile como o melhor classificado (GTCI, 2020).

Entre os principais problemas que os especialistas apontam para a baixa competitividade do Brasil estão a baixa capacidade de inovação da economia e sua deficiente qualificação de trabalhadores (DIÁLOGOS SETORIAIS UNIÃO EUROPEIA BRASIL, 2018). É nesta perspectiva que as novas Diretrizes Curriculares Nacionais (DCN's) para os cursos de engenharia, homologadas em 2019, estabelecem uma formação profissional mais alinhada com as demandas atuais. Além de fortalecer o conhecimento nas áreas tecnológica e social, as novas determinações buscam também a formação de profissionais capazes de liderar, inovar e administrar (ARANHA et al.,2017). Nesse sentido, a educação empreendedora adquire um importante papel no desenvolvimento dos novos engenheiros, uma vez que o comportamento, atitude e mentalidade empreendedora estão diretamente relacionadas com competências e habilidades de inovação, entre as quais destacam-se pensamento criativo, solução de problemas, trabalho em equipe, visão,quebra de paradigmas, liderança e networking, levando em consideração aspectos globais, políticos, econômicos, sociais, ambientais e culturais (ARANHA; DOS SANTOS; GARCIA, 2018).

Esse cenário, também oriundo do movimento de globalização, fez surgir alguns modelos de classificação, conhecidos como rankings (PERSPEKTYWY EDUCATION FOUNDATION, 2020; ROSS, 2019; QS WORLD UNIVERSITY RANKINGS, 2021; GEM GLOBAL, 2020). Na área educacional, alguns rankings classificam cursos e instituições de ensino superior em nível nacional e internacional tendo várias finalidades, entre elas, avaliar a qualidade destes, de acordo com diferentes critérios (ROSS, 2019; QS WORLD UNIVERSITY RANKINGS, 2021). Esses rankings são relevantes porque oferecem maior visibilidade para as instituições de ensino, conectam demanda e oferta educacional, ampliam as escolhas de formação profissional, preenchem necessidades do mercado de trabalho, entre outros. Dessa forma, fez-se necessário que as instituições de ensino superior do Brasil participassem de avaliações providas por rankings nacionais e internacionais, como uma forma de traçar estratégias de melhoria de ensino e pesquisa e ainda inserir-se em um contexto educacional de amplitude global. Como exemplo, a Universidade de São Paulo (USP); a Universidade de Campinas (Unicamp) e a Universidade Federal de Minas Gerais (UFMG) fizeram parte da análise do Times Higher Education/ THE e foram consideradas as melhores universidades brasileiras. Em relação 
aos cursos de engenharia do Brasil, segundo o ranking da consultoria britânica Quacquarelli Symonds (QS WORLD UNIVERSITY RANKINGS, 2021), a Universidade de São Paulo (USP), seguida da Universidade de Campinas (Unicamp) e Universidade Federal do Rio de Janeiro (UFRJ) foram apontadas como as melhores instituições na área de engenharia e tecnologia, pois ofertam ensino de excelência, com boa atuação em pesquisa e internacionalização.

Um importante instrumento de medição do empreendedorismo e suas características tem sido o Global Entrepreneurship Monitor (GEM), que consiste em um consórcio de equipes de pesquisadores na área de empreendedorismo vinculados a renomadas instituições acadêmicas e de pesquisa de mais de 100 países. É a única pesquisa em âmbito global que coleta dados sobre o empreendedorismo diretamente com os indivíduos empreendedores, o que possibilita a captura de dados sobre a economia informal, além das atividades econômicas formais, especialmente nos países de baixa e média renda (GEM BRASIL, 2020).

Apesar da existência desse panorama, há um gap na literatura brasileira de educação em engenharia acerca dos impactos desses rankings para as instituições e para o mercado. Um levantamento inicial realizado pelos autores sinaliza que na produção científica da Associação Brasileira de Educação nas Engenharias/ABENGE de 2015 a 2020, o estudo sobre rankings internacionais e ensino de engenharia está ausente, seja nos artigos publicados nos congressos anuais, nos livros sobre as sessões dirigidas e/ou na revista de Ensino da Engenharia, publicada pela ABENGE.

A ausência de artigos acadêmicos sobre os rankings, particularmente, relacionados aos cursos de graduação em engenharia emergem diversas questões que precisam de investigação. Nesta direção, a presente pesquisa é norteada pela seguinte questão básica: Quais são os requisitos essenciais que estão relacionados com os principais rankings internacionais e são capazes de explicitar a educação em engenharia nas universidades?

O objetivo desta pesquisa é duplo: a) identificar e explorar os requisitos que estão presentes nos principais rankings internacionais selecionados nesta pesquisa; b) analisar as interfaces entre os requisitos explorados e, com base nessas relações, propor um conjunto de requisitos essenciais.

O estudo está estruturado em seis seções. A primeira seção contém a introdução. $\mathrm{Na}$ segunda seção, está a revisão da literatura sobre os rankings internacionais definidos para esta pesquisa e, na terceira, estão mencionados os métodos e técnicas. Na quarta seção, são apresentados os requisitos essenciais analisados a partir das relações e interfaces encontradas. Para finalizar, são apresentados os resultados, as contribuições da pesquisa e as considerações finais na quinta e sexta seção.

\section{REVISÃO DE LITERATURA}

No sentido de atender os objetivos propostos, foi realizado um levantamento bibliográfico sobre quatro rankings internacionais, considerando modelos de classificação de cursos de graduação e empreendedorismo: QS World University Ranking (QS WORLD UNIVERSITY RANKINGS, 2021), Times Higher Education - THE (ROSS, 2019), European Ranking of Engineering Programs - EngiRank (PERSPEKTYWY EDUCATION FOUNDATION, 2020) e Global Entrepreneurship Monitor - GEM (GEM - GLOBAL, 2020). Em conjunto com as características destes modelos, são apresentados os quadros de requisitos que relacionam os principais aspectos que compõem cada um dos rankings estudados. Estes quadros também apresentam os graus de importância, dividido numa escala de três níveis, que foram atribuídos pelos autores a cada um dos pontos de acordo 
com sua relevância para a estruturação do modelo de classificação analisado com base nas pesquisas realizadas.

\subsection{QS World University Ranking}

A QS World University Rankings anual diferencia os ranking (quem é o melhor) de ratings - classificação (quem é bom e em quê). Segundo a QS, essa distinção é importante por dois motivos: a) as "classificações incluem apenas as 100 ou 1000 melhores universidades em todo o mundo, o que significa que as universidades mais novas têm dificuldade em competir com Harvards e Oxfords" ; b) uma classificação avalia as universidades sobre o desempenho delas em várias áreas, semelhantes às áreas consideradas em classificações, mas em vez de comparar as instituições umas com as outras, eles são julgados pelo seu desempenho em relação a somente um padrão estabelecido.

Segundo a QS Stars (QS WORLD UNIVERSITY RANKINGS, 2021), a importância de adotar a classificação proposta por ela é porque referencia os pontos fortes e fracos de uma universidade, criando evidências em dezenas de indicadores para a universidade receber uma pontuação geral (5 estrelas), além de uma pontuação para cada categoria. Não há limite para a quantidade de universidades que podem atingir a maior pontuação geral ou em qualquer categoria. Além dos critérios básicos, pode-se acrescentar critérios especializados como: gestão de hospitalidade e lazer, empregabilidade, diversidade, arte e cultura, responsabilidade social, inovação e inclusão.O Quadro 1 evidencia os requisitos que são necessários para que os indicadores do ranking sejam aprimorados em sua aplicabilidade.

Quadro 1- Principais requisitos e seu grau de importância para o projeto da QS

\begin{tabular}{|c|c|c|c|}
\hline \multirow{2}{*}{ Requisito } & \multicolumn{2}{c|}{ Grau de importância } \\
\cline { 2 - 4 } & Muito importante & Importante & Pouco importante \\
\hline Equipe Multidisciplinar e qualificada & $\mathrm{x}$ & & \\
\hline Rede de Parceiros & & $\mathrm{x}$ & \\
\hline Diversificação de fontes de recursos & & $\mathrm{x}$ & \\
\hline Amplitude geográfica & & $\mathrm{x}$ & \\
\hline $\begin{array}{c}\text { Utilização de indicadores globais como inovação } \\
\text { e ODS, empreendedorismo }\end{array}$ & & $\mathrm{x}$ & \\
\hline $\begin{array}{c}\text { Critérios de rankings para comparar e destacar } \\
\text { Metodologias de avaliação qualitativa e } \\
\text { quantitativa }\end{array}$ & & $\mathrm{x}$ & \\
\hline Mudanças após aplicação dos rankings nas & & & \\
\hline instituições & & $\mathrm{x}$ & \\
\hline Informações claras, completas e objetivas & $\mathrm{x}$ & & \\
\hline Destaque aos impactos do uso dos rankings & & & \\
\hline Diversificação de fontes de recursos & $\mathrm{x}$ & & \\
\hline
\end{tabular}

Fonte: Elaborado pelos autores.

\subsection{O Times Higher Education - THE}

O THE tem sido considerado um dos rankings de classificação educacional mais respeitados do mundo e se baseia no ensino, pesquisa, transferência de conhecimento e visão internacional (ROSS, 2019). São usados 13 indicadores para comparar a relação entre as universidades. Mesmo em caráter ainda experimental, esse ranking favorece uma agenda futura voltada para a conexão entre universidades, os 17 Objetivos de 
Desenvolvimento Sustentável (ODS) e suas 169 metas, com a valorização das políticas de extensão da universidade e seu impacto social, ambiental e cultural no território. Assim, o Quadro 2 relaciona os requisitos encontrados e seu grau de importância para o projeto do THE.

Quadro 2 - Principais requisitos e seu grau de importância para o projeto do THE

\begin{tabular}{|c|c|c|c|}
\hline \multirow{2}{*}{ Requisito } & \multicolumn{2}{|c|}{ Grau de importância } \\
\cline { 2 - 4 } & Muito importante & Importante & Pouco importante \\
\hline Rede de Parceiros & & $\mathrm{x}$ & \\
\hline Diversificação de fontes de recursos & $\mathrm{x}$ & $\mathrm{x}$ & \\
\hline $\begin{array}{c}\text { Utilização de indicadores globais como } \\
\text { inovação e ODS, empreendedorismo }\end{array}$ & & $\mathrm{x}$ & \\
\hline Metodologias de avaliação qualitativa & & $\mathrm{x}$ & \\
\hline $\begin{array}{c}\text { Mudanças pós aplicação dos rankings } \\
\text { nas instituições }\end{array}$ & & $\mathrm{x}$ & \\
\hline $\begin{array}{c}\text { Destaque a avaliação de impactos do } \\
\text { uso dos rankings referente as ODS }\end{array}$ & $\mathrm{x}$ & & \\
\hline $\begin{array}{c}\text { Adaptação de indicadores às questões } \\
\text { culturais e econômicas do país }\end{array}$ & & & \\
\hline
\end{tabular}

Fonte: Elaborado pelos autores.

\subsection{European Ranking of Engineering Programs - EngiRank}

Diferentemente dos mdelos anteriores, o EngiRank é centrado em cursos de engenharia. Este ranking busca preencher uma lacuna em relação às informações sobre ensino de engenharia na Europa; popularizar novos métodos de ensino; e atender as necessidades dos principais stakeholders. Ele é destinado, principalmente, a potenciais novos alunos do ensino superior e seus responsáveis; empregadores, na busca por novos talentos; e para auxiliar a gestão universitária (PERSPEKTYWY EDUCATION FOUNDATION, 2020). A primeira classificação dos programas de engenharia feita pelo EngiRank foi divulgada em 2020 como um projeto piloto. Esta versão englobou cursos e instituições de treze países (Bulgária, Croácia, Chipre, Estônia, Hungria, Letônia, Lituânia, Malta, Polônia, Romênia, Eslováquia, Eslovênia e República Tcheca) e foi desenvolvido pela Perspektywy Education Foundation e Foundation for the Development of the Education System com o apoio de várias entidades (PERSPEKTYWY EDUCATION FOUNDATION, 2020).

O projeto divulgou a classificação das instituições de ensino superior em um ranking geral e também "by subject" (por habilitação), de acordo com as diretrizes do Field of Science and Technology (FOS) mencionadas pela Organização para a Cooperação e Desenvolvimento Econômico (OCDE) , isto é, considerou-se as seguintes áreas: Chemical Engineering; Civil Engineering; Electrical, Electronic \& Information Engineering; Environmental Engineering; Materials Engineering Mechanical Engineering, Medical Engineering (PERSPEKTYWY EDUCATION FOUNDATION, 2020).

Com base no levantamento das informações sobre este ranking, o Quadro 3 apresenta os seus principais requisitos e os graus de importância de cada um destes. 
28 a 30 de SETEMBRO

Quadro 3 - Requisitos e graus de importância do EngiRank

\begin{tabular}{|c|c|c|c|}
\hline \multirow{2}{*}{ Requisitos } & \multicolumn{3}{|c|}{ Grau de importância } \\
\hline & Muito importante & Importante & Pouco importante \\
\hline Transparência & $\mathrm{x}$ & & \\
\hline Metodologia bem definida & $\mathrm{x}$ & & \\
\hline Propósito bem definido & $\mathrm{x}$ & & \\
\hline Indicadores bem definidos & $x$ & & \\
\hline $\begin{array}{l}\text { Fontes de informação e procedimentos de } \\
\text { coleta bem definidos }\end{array}$ & $\mathrm{x}$ & & \\
\hline Representatividade geográfica & $\mathrm{x}$ & & \\
\hline Considerar contexto regional & $\mathrm{x}$ & & \\
\hline Ferramentas de gestão & & $x$ & \\
\hline Equipe multidisciplinar & & $\mathrm{x}$ & \\
\hline Recursos financeiros & $\mathrm{x}$ & & \\
\hline $\begin{array}{l}\text { Disponibilidade de tempo e energia } \\
\text { (pessoas) }\end{array}$ & $\mathrm{x}$ & & \\
\hline Conhecer outras iniciativas / boas práticas & $\mathrm{x}$ & & \\
\hline Revisão contínua & & $\mathrm{x}$ & \\
\hline
\end{tabular}

Fonte: Elaborado pelos autores.

\subsection{Global Entrepreneurship Monitor - GEM}

Enquanto os modelos anteriores avaliam ensino superior, o GEM é focado na atividade empreendedora da sociedade. O GEM foi fundado pela London Business School (LBS) e Babson College em 1997 na LBS pelos professores de Empreendedorismo, Bill Bygrave e Michael Hay. Com o apoio de George Bain, reitor da LBS na época, Michael e Bill idealizaram sobre o que seria necessário para criar um índice para competitividade empresarial semelhante ao Índice de Competitividade Global que é publicado anualmente pelo Fórum Econômico Mundial (GEM - GLOBAL, 2020). O GEM promove a inovação para explorar novas oportunidades, impulsionar a produtividade e criar empregos, também abordando alguns dos desafios mais difíceis da sociedade, como a conquista dos ODS e a Agenda 2030 (GEM - BRASIL, 2020).

A estrutura conceitual do GEM é determinada por valores sociais e atributos individuais, valor agregado e empregos. Entretanto, a estrutura também considera o contexto social, cultural, político e econômico, que tanto influencia como é influenciado pelo empreendedorismo. O GEM consiste em duas pesquisas: a) Pesquisa com a População Adulta (Adult Population Survey - APS), que consiste em um levantamento domiciliar junto a uma amostra representativa de pessoas com idade entre 18 e 64 anos de cada país participante do GEM ; b) Pesquisa com Especialistas Nacionais (National Expert Survey $N E S$ ), em cada um dos países participantes são entrevistados no mínimo 36 especialistas do setor público e privado como: agentes de promoção de políticas públicas, acadêmicos, empreendedores e demais pessoas que atuam e conhecem 0 contexto do empreendedorismo local (GEM - BRASIL, 2020).

A rigorosa abordagem metodológica do GEM transforma as respostas obtidas nas pesquisas em medidas precisas do nível de atividade empreendedora, fornecendo dados relevantes para que os formuladores de políticas possam monitorar e avaliar os impactos das políticas e iniciativas. No quadro a seguir, são apresentados um conjunto de fatores considerados importantes para a elaboração de matriz de critérios para este ranking. 
Quadro 4 - Matriz de Conjunto de Critérios para o Ranking.

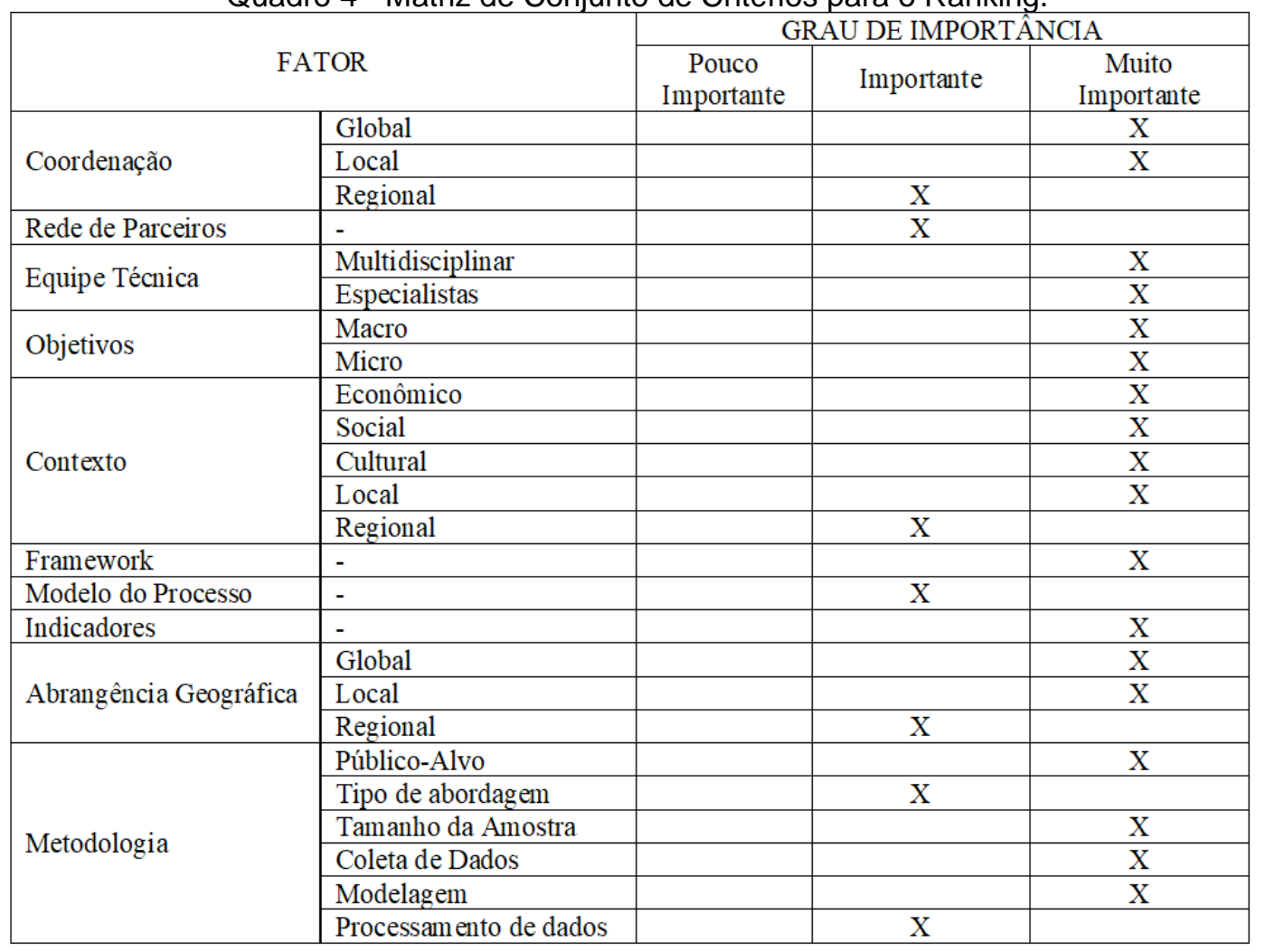

Fonte: Elaborado pelos autores

\subsection{Quadro Geral}

A partir do estudo individual de cada ranking apresentado, é possível verificar a existência de conexões e interfaces entre as classificações, mas também aspectos que os diferenciam. O Quadro 5 demonstra o panorama relacional que pode ser estabelecido e os graus de importância atribuídos a cada critério.

Através do Quadro 5, nota-se que a maioria dos critérios são comuns aos quatro rankings estudados, mesmo que a relação tenha considerado modelos que avaliam objetos diferentes, como os rankings voltados especificamente para instituições de ensino e o GEM, que é focado no empreendedorismo. Também é possível perceber que, para os pesquisadores, nenhum dos requisitos pode ser considerado de pouca importância, pois, de fato, sistematizam o funcionamento dos modelos estudados. 
28 a 30 de SETEMBRO

Quadro 5 - Requisitos e graus de importância dos rankings estudados

\begin{tabular}{|c|c|c|}
\hline \multicolumn{3}{|c|}{ REQUISITQS } \\
\hline EnqiRank & GEM & QS / THE \\
\hline Transparência & Informações claras, completas e objetivas & Informações claras, completas e objetivas \\
\hline Propósito bem definido & Dbjetivos (micro e macro) & \\
\hline \multicolumn{3}{|l|}{$\begin{array}{l}\text { Fontes de informação e } \\
\text { procedimento de coleta }\end{array}$} \\
\hline Metodologia & $\begin{array}{l}\text { Metodologia (público-alvo, tipo de abordagem, } \\
\text { tamanho da amostra, coleta de dados, modelagem, } \\
\text { processamento de dados) }\end{array}$ & $\begin{array}{l}\text { Metodologias de avaliação qualitativa e } \\
\text { quantitativa }\end{array}$ \\
\hline Indicadores bem definidos & Indicadores & $\begin{array}{l}\text { Utilização de indicadores globais como } \\
\text { inovação e DDS, empreendedorismo }\end{array}$ \\
\hline Contexto regional & Contexto [ecônomica, social, cultural, local, regional] & $\begin{array}{l}\text { Adaptação de indicadores às questões } \\
\text { culturais e econômicas do pais }\end{array}$ \\
\hline Representatividade geográfica & Abrangência geográfica (global, local, regional) & Amplitude geográfica \\
\hline \multicolumn{3}{|l|}{$\begin{array}{l}\text { Apresentação de informações de } \\
\text { forma compreensivel }\end{array}$} \\
\hline Equipe multidisciplinar & Equipe técnica (multidisciplinar e especialistas) & Equipe multidisciplinar e qualificada \\
\hline \multicolumn{3}{|l|}{$\begin{array}{c}\text { Disponibilidade de tempo e } \\
\text { energia (pessoas) }\end{array}$} \\
\hline & Coordenação (global, regional, local) & \\
\hline \multirow[t]{2}{*}{ Recursos financeiros } & Patrocinadores e rede de parceiros & Diversificação de fontes de recursos \\
\hline & Patrocinadores e rede de parceiros & Rede de parceiros \\
\hline Revisão contínua & & $\begin{array}{c}\text { Mudanças após aplicação dos rankings } \\
\text { nas instituições }\end{array}$ \\
\hline \multirow[t]{2}{*}{$\begin{array}{l}\text { Conhecer outras iniciativas i boas } \\
\text { práticas }\end{array}$} & & $\begin{array}{c}\text { Critérios de rankings para comparar e } \\
\text { destacar }\end{array}$ \\
\hline & Framework & \\
\hline \multirow[t]{2}{*}{ Ferramentas de gestão } & Ferramentas de gestão & \\
\hline & $\begin{array}{l}\text { Conquista dos Dbjetivos de Desenvolvimento } \\
\text { Sustentável [DDS] }\end{array}$ & $\begin{array}{l}\text { Destaque a avaliação de impactos do uso } \\
\text { dos rankings referente as QDS }\end{array}$ \\
\hline
\end{tabular}

Grau de importância

Muito importante

Importante

Pouco importante Vários

Fonte: Elaborado pelos autores.

\section{MATERIAIS E MÉTODOS}

O método de pesquisa utilizado para o desenvolvimento deste estudo pode ser caracterizado como sendo de natureza exploratória, descritiva e explicativa (GIL, 2002).

Sob o ponto de vista dos objetivos, a pesquisa é exploratória, pois envolve um levantamento bibliográfico a partir da literatura especializada; explicativa, devido ao foco na exploração de critérios considerados importantes, na percepção dos pesquisadores, para elaboração de critérios essenciais existentes entre os rankings analisados. Gil (2008) descreve que um estudo exploratório tem por objetivo proporcionar uma visão geral, do tipo aproximativo, acerca de determinado fato, podendo também ser realizado quando o tema em estudo é pouco explorado e torna-se difícil formular hipóteses precisas e operacionalizáveis, o que é uma realidade, quando se trata de avaliar questões sobre rankings internacionais.

O presente estudo surge no âmbito do grupo de pesquisa de Dinâmicas Empreendedoras/ NPDE da Universidade Federal de Itajubá, com a ideia de acompanhar o desenvolvimento da educação empreendedora nos cursos de engenharia brasileiros em função das determinações da nova DCN. Em função da lacuna encontrada na literatura, os pesquisadores do presente estudo realizaram um levantamento dos principais rankings existentes que considerassem empreendedorismo e cursos de nível superior, em especial, 
cursos de engenharia. Através dessa busca, emergiram quatro rankings: QS World University Rankings (QS); Times Higher Education World University Rankings (THE); European Ranking of Engineering Programs (EngiRank); e o Global Entrepreneurship Monitor (GEM). A partir daí, estabeleceu-se uma investigação sobre cada um destes modelos de classificação a fim de conhecer melhor suas características, objetivos, metodologias e seus organizadores. Estas informações permitiram relacionar os principais critérios que estruturam estes rankings e atribuir níveis de importância a cada um destes requisitos. Os graus de importância foram divididos em três posições (muito importante, importante e pouco importante) e determinados pelos pesquisadores em função da profunda análise realizada, o que permitiu verificar as principais diferenças e similaridades nas concepções de cada modelo. Todos esses dados foram organizados em quadros por ranking que, posteriormente, foram consolidados e harmonizados pelos pesquisadores, dando origem à proposta de um conjunto de requisitos essenciais para um modelo de classificação. A Figura 1 sistematiza estas etapas de pesquisa:

Figura 1: Fluxograma de etapas principais da pesquisa

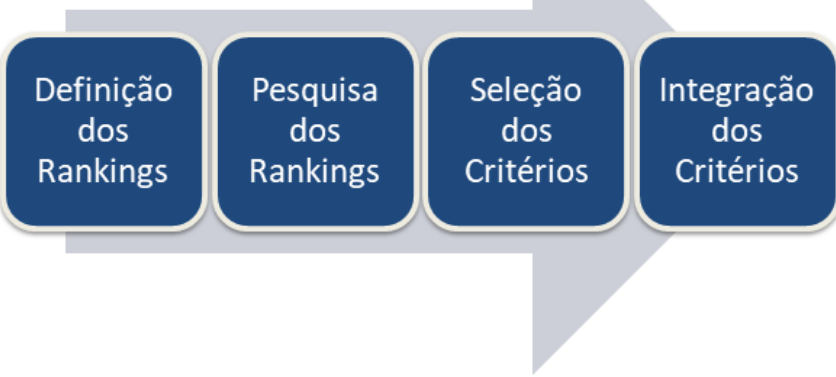

. Fonte: Elaborado pelos autores

- Definição dos rankings: Foram selecionados 4 rankings - QS World University Rankings (QS); Times Higher Education World University Rankings (THE); European Ranking of Engineering Programs (EngiRank); e o Global Entrepreneurship Monitor (GEM) - distribuídos aleatoriamente para cada um dos pesquisadores;

- Pesquisa dos rankings: Teve como finalidade investigar o ranking para conhecer melhor cada um dos modelos;

- Seleção dos critérios de cada ranking: Com base na pesquisa realizada, cada pesquisador identificou os fatores que dão estruturação aos modelos e podem ser considerados mais importantes. Para isso, foi estabelecido uma escala de três níveis: muito importante, importante e pouco importante.

- Integração dos critérios: A equipe consolidou e harmonizou os dados dos critérios de cada ranking pesquisado; na sequência, fez a proposição dos requisitos essenciais.

\section{PROPOSTA DE REQUISITOS ESSENCIAIS}

Tendo como base as pesquisas realizadas e mencionadas neste trabalho, é possível chegar a uma proposta de requisitos essenciais a partir da integração dos diferentes modelos, conforme visto no Quadro 5. A seguir, a Figura 2 apresenta a proposta considerada. 
Figura 2 - Proposta de requisitos essenciais de acordo com os rankings analisados

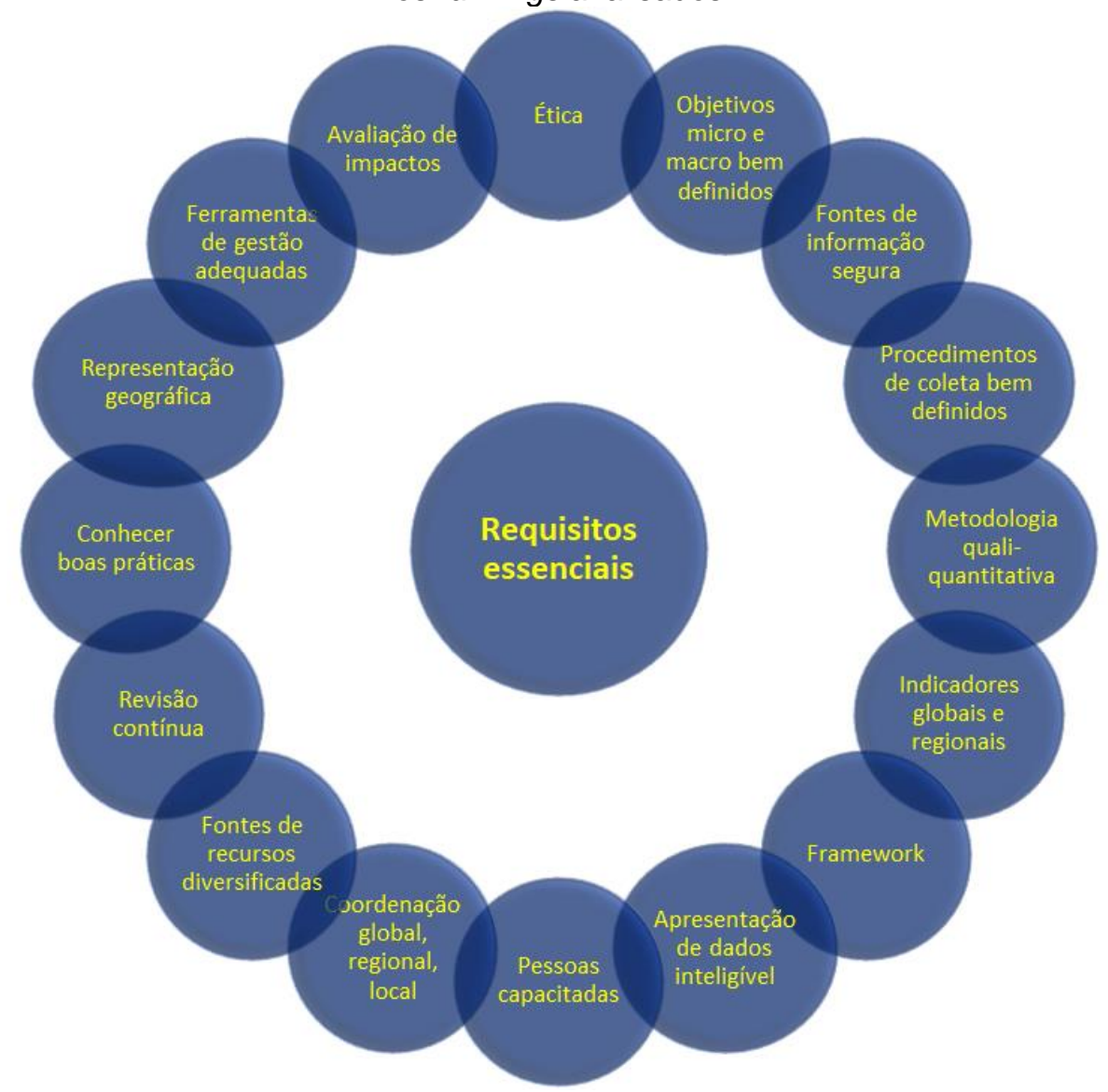

Fonte: Elaborado pelos autores.

A proposta de requisitos da Figura 2 baseia-se na ideia de que é fundamental possuir objetivos micro e macro bem delimitados, reconhecendo a diversidade das diferentes entidades a serem avaliadas, bem como as suas distintas missões e objetivos. Já as fontes de informação e os procedimentos de coleta devem ser bem definidos e adequados aos dados que se deseja coletar, além da necessidade de conhecer bem o significado destas informações a fim de não gerar diferenças na interpretação dos dados.

A relevância da metodologia quali-quantitativa tem como fundamento combinar diferentes perspectivas a fim de gerar uma visão mais completa do objeto avaliado. Para isso, é importante ter procedimentos metodológicos precisos que possam ser sistematizados em um modelo adequado ao tamanho da amostra e que considerem o processamento e revisão sistemática dos dados a fim de minimizar a ocorrência de erros. Aliado ao aspecto metodológico, está a relação de indicadores que deve considerar as diferenças culturais e econômicas referendadas pelos índices das ODS, refletindo a respeito das diferenças políticas, institucionais, econômicas, culturais, demográficas e de empreendedorismo e inovação; até porque é essencial que o modelo possa ser caracterizado por uma representatividade geográfica. Neste sentido, importante atenção deve ser dada à validade dos indicadores escolhidos, aos pesos atribuídos a estes e às necessidades de alterações, afinal estas questões é que darão elevada sistematização ao resultado final. Aliás, é fundamental que esta conclusão e divulgação de informações seja representada por clareza e facilidade de interpretação a fim de evitar dubiedades, oportunizando a acessibilidade dos resultados ao devido público a que se destina. 
É relevante perceber que todo este processo precisa de uma diversificação de fonte de recursos e parceiros; equipes com disponibilidade, energia e capacitação em nível local, regional e global, bem como ferramentas e softwares adequados a cada etapa. Ainda é necessário salientar que o processo não termina na divulgação de resultados, pois a análise dos impactos da avaliação e dos indicadores utilizados é importante para verificar se os objetivos definidos estão sendo atingidos e se o modelo é significativo para o aprimoramento das entidades avaliadas. Por isso, é necessário desenvolver um framework que permita a realização de revisões contínuas visando garantir a qualidade de todo o processo também pautado pelo conhecimento de boas práticas, de outras iniciativas existentes e pela ética, concedendo transparência a todas as etapas. Dessa forma, a proposta de requisitos essenciais apresentada considera os aspectos em comum dos rankings estudados, mas também os pontos que os diferenciam, fazendo com que se tenha uma visão mais ampliada do objeto a ser avaliado e apresentando resultados que possam aperfeiçoar mais o trabalho das instituições analisadas.

\section{RESULTADOS E CONTRIBUIÇÕES DA PESQUISA}

Este estudo amplia a possibilidade de critérios de classificação a partir de modelos já existentes e, por isso, pode auxiliar o aprimoramento de rankings a nível global ao servir de base para analisar as universidades em relação a aspectos desprezados em variados rankings. Assim, entre os resultados da pesquisa, é possível destacar: o preenchimento da lacuna existente na literatura sobre estudos de rankings internacionais com foco na educação em engenharia; orientação aos gestores educacionais em relação a tomada de decisão no processo de planejamento estratégico das instituições; favorecimento do equilíbrio entre indicadores e evidência das diferenças entre as classificações; e o auxílio para a construção de novos modelos de classificação.

Por outro lado, é necessário apontar que não foram encontrados aspectos que considerem as especificidades dos cursos de engenharia. Além disso, percebe-se que, apesar da reconhecida importância da educação empreendedora (FAYOLLE; GAILLY, 2008), não foi possível identificá-la como requisito essencial nos rankings analisados, demonstrando que, apesar dos diferentes objetivos e dos distintos objetos a serem avaliados em cada ranking, parece haver uma base comum de critérios entre os modelos de classificação. Dessa forma, a pesquisa também contribui para a necessidade de fomentar novas investigações a fim de entender o motivo pelo qual a educação empreendedora é desconsiderada e para o desenvolvimento de propostas que possam melhor avaliar as condições mais específicas de cursos de engenharia, agregando a perspectiva da educação empreendedora.

\section{CONSIDERAÇÕES FINAIS}

É possível dizer que os resultados encontrados são inovadores. Os objetivos da pesquisa foram plenamente atingidos e contribuem para preencher o gap identificado na literatura brasileira do campo de educação em engenharia, respondendo à questão básica formulada. Desse modo, a proposta de requisitos essenciais apresentada por este estudo inova ao fornecer uma maior dimensão de análise ao combinar os aspectos em comum dos rankings, mas também os pontos que os diferenciam.

Os resultados ainda produzem diversas implicações práticas. Serão destacadas duas: Primeiro, o apoio ao planejamento de cursos de graduação. É relevante pesquisar e 
conhecer os distintos modelos para a criação e aprimoramento dos rankings e, principalmente, para apoiar o planejamento dos cursos de graduação brasileiros em relação ao panorama internacional, até porque muitas instituições nacionais já são avaliadas por estas classificações. Segundo, o fomento de novas discussões entre coordenadores, diretores e professores. Os resultados podem servir de base para o desenvolvimento de outras análises a fim de trazer novas discussões, em especial, que considerem mais especificamente os cursos de engenharia.Os resultados encontrados evidenciam que, apesar da existência de diferentes rankings com variados objetivos e distintos objetos a serem avaliados, parece haver uma base comum de requisitos entre eles.

\section{REFERÊNCIAS}

ARANHA, E.A.; DOS SANTOS, P. H.; GARCIA, N.A.P. EDLE: an integrated tool to foster entrepreneurial skills development in engineering education. Educational Technology Research and development, vol. 66, № 6, 2018,pág.1571-1599.

DIÁLOGOS SETORIAIS UNIÃO EUROPEIA - BRASIL. Estudo comparado sobre os currículos dos cursos de engenharia no Brasil e na Europa e sugestões para o fomento à inovação, 2018. http://www.sectordialogues.org/sites/default/files/publicacao_educ-30mar-web-final.pdf. Acesso em: 15 abr. 2021.

FAYOLLE, A; GAILLY, B. From craft to science: teaching models and learning processes in entrepreneurship education. Journal of European Industrial Training, vol.32, № 7, 2008, pág.569-593

FINNVEDEN, G. Universities must walk the talk on sustainable development. Times Higher Education Blog, 2019. Disponível em: https://www.timeshighereducation.com/worlduniversity-rankings/universities-must-walk-talk-sustainable-development. Acesso em: 15 abr. 2021.

GEM - BRASIL. Global Entrepreneurship Monitor Empreendedorismo no Brasil: 2019 $\backslash$ Coordenação de Simara Maria de Souza Silveira Greco; diversos autores -- Curitiba: IBQP, 2020

GEM - GLOBAL. Global Entrepreneurship Research Association, London Business School, Regents Park, London NW1 4SA, UK. 2019/2020. ISBN (print): 978-1-9160178-25

GIL, A. C. Como Elaborar Projetos de Pesquisa. 4a Edição. São Paulo: Atlas, 2002.

GIL, A. C. Métodos e Técnicas de Pesquisa Social. 6a Edição. São Paulo: Atlas, 2008.

GTCI - GLOBAL TALENT COMPETITIVENESS INDEX 2020. Global Talent in the Age of Artificial Intelligence. Insead and Google. 2020.

OLIVEIRA, V. F. de; ALMEIDA, N. N. de; CARVALHO, D. M. de; PEREIRA, F. A. A. Um estudo sobre a expansão da formação em engenharia no Brasil. Revista de Ensino de Engenharia, [s. I.], v. 32, n. 3, 2013 
PERSPEKTYWY EDUCATION FOUNDATION. Report EngiRank - Pilot Edition. European Ranking of Engineering Programs, 2020. Disponível em: http://engirank.eu/wp-content/uploads/2020/12/Engi-Rank-2020-12-09.pdf. Acesso em: 10 fev. 2021.

QS WORLD UNIVERSITY RANKINGS. What is the QS World University Rankings?, 2021.Disponível em: https://www.qs.com/rankings/. Acesso em: 15 abr. 2021

ROSS, D. We're including all 17 SDG in the 2020 in the University Impact Rankings. THE World University Rankings, 2019. Disponível em: https://www.timeshighereducation.com/blog/were-including-all-17-sdgs-2020-universityimpact-rankings. Acesso em: 15 abr. 2021

SILVA, Q. F. da; SANTOS, R. dos; SOUZA, I. A. M. de. A importância da educação continuada após a graduação em engenharia e suas conexões com as tendências do mercado de trabalho. In: VII Congresso Brasileiro de Educação em Engenharia e II Simpósio Internacional de Educação em Engenharia da Abenge, 2019, Fortaleza. Anais. Fortaleza. Disponível em:

ttp://www.abenge.org.br/sis_artigo_doi.php?e=COBENGE\&a=19\&c=1998. Acesso em: 15 abr. 2021

\section{ESSENTIAL REQUIREMENTS PROPOSAL BASED ON INTERNATIONAL HIGHER EDUCATION AND ENTREPRENEURSHIP RANKINGS}

Abstract: The demands placed on engineers are changing. For this reason, the 2019 National Curriculum Guidelines establish training that is more in line with current demands and give more relevance to entrepreneurial education in Brazilian courses. This scenario, also from the globalization movement, gave rise to some classification models known as rankings, including for education and entrepreneurship. However, there is a gap in the Brazilian engineering education literature on this subject that raises several issues. In this sense, the present research is guided by: What are the essential requirements that are related to the main international rankings and are able to explain engineering education in universities? The research objective is twofold: a) to identify and explore the requirements that are present in the main international rankings selected; b) analyze the interfaces between the requirements explored and, based on these relationships, propose a set of essential requirements. For this, an exploratory, descriptive and explanatory research was carried out considering four international rankings. Based on the investigation of these models, it was possible to come up with a proposal for essential requirements. Thus, this study innovates by presenting a model that provides a greater dimension of analysis and, on the other hand, points out that it was not possible to identify entrepreneurial education as an essential requirement in the analyzed rankings. The results found show that, despite the existence of different rankings with different objectives and objects to be evaluated, there seems to be a common base of requirements among them.

Keywords: Engineering Education; Rankings; Entrepreneurial Education; Higher Education; Assessment 